\title{
The concordance of endoscopic and histologic findings of 530 pediatric patients suspicious for celiac disease in a referral center, Isfahan, Iran
}

\author{
Azar Naimi $^{1^{\circledR}}$, Azar Baradaran $^{1}{ }^{\circledR}$, Elahe Pirpiran $^{1}{ }^{\mathbb{D}}$, Peiman Nasri $^{(\mathbb{D}}$, Masoud Akhlaghi $^{1 *}$ \\ ${ }^{1}$ Department of Pathology, School of Medicine, Isfahan University of Medical Sciences, Isfahan, Iran \\ ${ }^{2}$ Department of Pediatrics, Isfahan University of Medical Sciences, Isfahan, Iran
}

\section{Correspondence to: Masoud Akhlaghi, Email: masoued71@gmail.com \\ Received: 3 Nov. 2020 Accepted: 24 Dec. 2020 ePublished: 29 Dec. 2020}

Keywords: Endoscopy, Celiac disease, Children

\begin{abstract}
Introduction: The purpose of this study is evaluation of concordance of histologic and endoscopic findings in pediatrics referred to Imam Hossein children's hospital, in Isfahan for evaluation of celiac disease. An accurate prediction of the likelihood of a histologic finding in the setting of a visually normal endoscopy can help prepare families for unanticipated results.

Patients and Methods: This is a retrospective study conducted from 2015-2017. The sampling method is census and all eligible patients are included in the study. At the beginning of the work, obtaining consent for entering the project has been done through interviews. After that, patients information such as file number, age and gender according to the file were entered into the data gathering sheet. Data were analyzed using SPSS software version 22. Pvalue $<0.05$ was considered significant.

Results: Around 530 patients suspected of having celiac disease participated in the present study. The mean age of the subjects was $6.88 \pm 4.31$ years. Among them, $57.6 \%$ (305 patients) were girls and $42.4 \%$ (225 patients) were boys. According to the results, the kappa agreement coefficient is equal to 0.42 . There was moderate agreement between endoscopy and pathology findings (kappa $=0.42$ ).

Conclusion: The results of the study and the kappa Cohen coefficient indicate the appropriateness of using endoscopy in patients with suspected celiac disease.
\end{abstract}

\section{Introduction}

Celiac disease is an autoimmune disease, in genetically predisposed people, by the ingestion of proteins loaded in proline and glutamine. It happens in individuals with a mean prevalence of around $1 \%$ of the population. Celiac disease is detected by an inflammatory consequence, primarily in the upper small intestine, with aspects of infiltration of the lamina propria and also beneath the epithelial cells which results in chronic inflammatory condition and progressive villous atrophy $(1,2)$.

Recently the task of serology is becoming more and more crucial, hence, regarding the guidelines of European Society of Pediatric Gastroenterology, Hepatology, and Nutrition, the identification of celiac disease can be conducted without histology in a group of patients with particular conditions like children, high titers of antitissue transglutaminase antibodies, presence of human leukocyte antigen-DQ2, and the positivity of anti-endomysial antibodies (3). Nevertheless, existing guidelines point

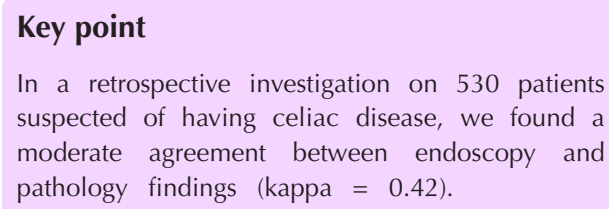

out histological examination as the gold standard for the detection of celiac disease. The morphologic features of this disease are crypt hyperplasia, villous atrophy, infiltration of the superficial epithelium according to the Marsh classification and its modifications (4-8). Therefore, pathological affirmation of these typical small bowel alterations is at present accepted as the gold standard to determine the diagnosis of celiac disease in individuals with positive antibody titer. To conduct an exact diagnosis, the biopsy fragments have to be well-oriented with good quality. In fact, around 4 to 6 duodenal biopsies, counting a bulb biopsy, are necessary to present the diagnosis of celiac disease (7,9-13).

Therefore, the affirmation of celiac 
disease can also be neglect if the disorder is not supposed and biopsy sampling not handled. Therefore, in these conditions, the role of the endoscopist is important, due to the significant impact of the macroscopic feature of the duodenum (14-16).

\section{Objectives}

Due to the above issues and the increasing number of patients with celiac disease, a comprehensive study with a higher sample size and a more standard method is necessary.

Therefore, the purpose of this study is evaluation of concordance of histologic and endoscopic features in pediatrics suspicious for celiac disease who referred to an educational hospital in Isfahan.

\section{Patients and Methods}

Study design

This was a retrospective study conducted at Imam Hossein hospital from 2015-2017.

\section{Inclusion and exclusion criteria}

Inclusion criteria: Pediatric patients clinically suspicious for celiac disease, age between one month and 18 years.

Exclusion criteria: Having a defect in the file information, existence of other gastrointestinal diseases, and history of intervention that has changed the intestinal tissue.

\section{Sampling method and sample size}

The sampling method is census and all eligible patients were included in the study.

\section{Data collection}

At the beginning of the work, obtaining consent to enter the project has been done through interviews by the project executors. After that, for all patients suspicious of celiac disease, patients' information such as file number, age and gender according to the file were entered into the questionnaire. In celiac disease, if the results of blood tests show celiac disease, the patient undergoes an endoscopy to evaluate the small intestine and remove small tissue samples (biopsies). The sample is examined in a laboratory to determine the histologic status.

After collecting the necessary information, the results of histology and endoscopy in patients have been evaluated and compared. Positive histologic findings were classified according to the Marsh classification; Marsh I; increased number of intraepithelial lymphocytes (IELs) with normal mucosa architecture; Marsh II; proliferation of the crypts and increased number of IELs and Marsh III; destructive lesion including partial or complete villous atrophy and crypt hypertrophy with increased number of IELs. In cases who the pathology report is in favor of celiac disease but the diagnosis is not definitive, the status of their disease was determined by contacting patients.

\section{Ethical issues}

The research followed the tenets of the Declaration of Helsinki. The institutional ethical committee at Isfahan University of Medical Sciences approved all study protocols (IR.MUI.MED.REC.7397.231). Accordingly, written informed consent was taken from all participants before any intervention.

\section{Statistical analysis}

The collected data were imported into SPSS software version 22 and analyzed with appropriate statistical tests. The significance level in the present study was less than 0.05 .

\section{Results}

Around 530 patients suspected of having celiac disease participated in the present study. The mean age of the subjects was $6.88 \pm 4.31$ years. Among them, 57.5\% (305 patients) are girls and $42.4 \%$ (225 patients) are boys.

Based on endoscopic results, 24.1\% (128 patients) of patients with suspected celiac disease were positive (reveal atrophy or scalloping) and $75.8 \%$ (402 patients) of patients with suspected celiac disease were negative. Additionally, based on the pathological results, $11.9 \%$ (63 patients) of patients with suspected celiac disease were positive (intraepithelial lymphocytosis and/or crypt hyperplasia and villi atrophy) and $88.1 \%$ (467 patients) of patients with suspected celiac disease were negative.

In group of positive histologic findings, 50 patients had Marsh 3 (10.5\%), 6 patients Marsh 2 (1.2\%) and 7 patients Marsh 1 (1.4\%) of celiac disease.

Table 1 shows the agreement of Cohen's kappa coefficient based on endoscopic and pathological results. In addition, sensitivity and specificity of endoscopic findings for celiac disease, are reported in this table. According to the results of the table, the kappa agreement coefficient is equal to 0.42 .

Table 1. p Kappa agreement coefficient and sensitivity and specificity

\begin{tabular}{|c|c|c|c|c|c|c|c|c|c|}
\hline & & \multicolumn{2}{|l|}{ Pathology } & \multirow{2}{*}{$\begin{array}{c}\text { Kappa agreement } \\
\text { coefficient }\end{array}$} & \multirow{2}{*}{ Sensitivity } & \multirow{2}{*}{ Specificity } & \multirow{2}{*}{ PPV } & \multirow{2}{*}{ NPV } & \multirow{2}{*}{$P$ value } \\
\hline & & Positive & Negative & & & & & & \\
\hline \multirow{2}{*}{ Endoscopy } & Positive & $49(38.3 \%)$ & $79(61.7 \%)$ & \multirow{2}{*}{0.42} & \multirow{2}{*}{0.73} & \multirow{2}{*}{0.83} & \multirow{2}{*}{0.38} & \multirow{2}{*}{0.96} & \multirow{2}{*}{$<0.001$} \\
\hline & Negative & $14(3.5 \%)$ & $388(96.5 \%)$ & & & & & & \\
\hline
\end{tabular}

PPV, positive predictive value; NPV, Negative predictive value. 


\section{Discussion}

Celiac disease is an autoimmune disease with significant prevalence in the general population that can manifest at any age and shows a rather varied clinical presentation. Due to the large number of patients with nonspecific gastrointestinal and extra-intestinal symptoms or even asymptomatic, diagnosis is often challenging (17). Currently, an increase in the prevalence of the disease in its atypical manifestations is well known and less than one third of adult patients present chronic diarrhea $(18,19)$. Therefore, the aim of this study was to determine the agreement between endoscopic and pathological findings in patients with suspected celiac disease and to predict the likelihood of a positive histologic finding in the setting of a visually normal endoscopy.

The final result of the study showed that the agreement between the endoscopic and pathological findings was equal to 0.42 . Besides, the sensitivity is equal to 0.68 and the specificity is equal to 0.76 .

In follow up all the patients with positive histologic findings, had elevated serum transglutaminase antibody (tTG) and the diagnosis of Celiac, confirmed for them.

In a study by Kar et al, the correlation between endoscopic and histological findings was $66 \%$ in 400 patients.

Other studies in adults have suggested that routine duodenal biopsies be performed (20). Previous pediatric studies have supported routine biopsy in endoscopy. Studies in children have reported a mismatch between endoscopic and histological results.

In another study of 94 patients, the sensitivity and specificity of duodenal endoscopy were $82 \%$ and $27 \%$, respectively, and gastric trunk endoscopy was $57 \%$ and $47 \%$ sensitive and specific (21).

In another study in Italy, endoscopy often provided inaccurate results with less deterioration than biopsy (22). Oderda et al found a $13.8 \%$ correlation between 32 biopsy responses and their endoscopic responses (23).

\section{Conclusion}

Based on the results of the study the amount of agreement between the endoscopic and pathological findings was equal to 0.44 . The kappa Cohen coefficient indicates the appropriateness of using endoscopy in individuals with supposed celiac disease.

\section{Limitations of the study}

One of the limitations of the present study was the small sample size. It is suggested to use more sample size in other studies and also to use clinical trial and or follow-up studies.

\section{Authors' contribution}

$M A$ and $A N$ and $A B$ were the principal investigators of the study. MA and $\mathrm{AN}$ were included in preparing the concept and design. EP and AN revisited the manuscript and critically evaluated the intellectual contents. All authors participated in preparing the final draft of the manuscript, revised the manuscript and critically evaluated the intellectual contents. All authors have read and approved the content of the manuscript and confirmed the accuracy or integrity of any part of the work.

\section{Ethical considerations}

Ethical issues (including plagiarism, data fabrication, double publication) have been completely observed by the authors.

\section{Conflicts of interest}

The authors declare that they have no competing interests.

Funding/Support

None.

\section{References}

1. Di Sabatino A, Corazza GR. Coeliac disease. Lancet 2009;373:1480-93. doi: 10.1016/S0140-6736(09)60254-3.

2. Green PH, Cellier C. Celiac disease. N Engl J Med. 2007;357:1731-43. doi: 10.1056/NEJMra071600.

3. Husby S, Koletzko S, Korponay-Szabó IR, Mearin ML, Phillips A, Shamir R, et al. European Society for Pediatric Gastroenterology, Hepatology, and Nutrition guidelines for the diagnosis of coeliac disease. J Pediatr Gastroenterol Nutr. 2012;54:136-60. doi: 10.1097/MPG.0b013e31821a23d0.

4. Rostom A, MurrayJA, KagnoffMF. American Gastroenterological Association (AGA) Institute technical review on the diagnosis and management of celiac disease. Gastroenterology. 2006;131:1981-2002. doi: 10.1053/j.gastro.2006.10.004.

5. Marsh MN. Gluten, major histocompatibility complex, and the small intestine. A molecular and immunobiologic approach to the spectrum of gluten sensitivity ('celiac sprue') Gastroenterology. 1992;102:330-54.

6. Oberhuber G, Granditsch G, Vogelsang H. The histopathology of coeliac disease: time for a standardized report scheme for pathologists. Eur J Gastroenterol Hepatol. 1999;11:1185-94. doi: 10.1097/00042737-199910000-00019.

7. Vogelsang H, Hänel S, Steiner B, Oberhuber G. Diagnostic duodenal bulb biopsy in celiac disease. Endoscopy. 2001;33:336-40. doi: 10.1055/s-2001-13702.

8. United European Gastroenterology. When is a coeliac a coeliac? Report of a working group of the United European Gastroenterology Week in Amsterdam, 2001. Eur J Gastroenterol Hepatol. 2001;13:1123-8. doi: 10.1097/00042737-200109000-00023.

9. Hopper AD, Cross SS, Sanders DS. Patchy villous atrophy in adult patients with suspected gluten-sensitive enteropathy: is a multiple duodenal biopsy strategy appropriate? Endoscopy. 2008;40:219-24. doi: 10.1055/s-2007-995361.

10. Brocchi E, Corazza GR, Brusco G, Mangia L, Gasbarrini G. Unsuspected celiac disease diagnosed by endoscopic visualization of duodenal bulb micronodules. Gastrointest Endosc. 1996;44:610-1. doi: 10.1016/s0016-5107(96)700202.

11. Cammarota G, Cesaro P, La Mura R, Martino A, Cazzato A, Miele L, et al. Role of the "immersion technique" in diagnosing celiac disease with villous atrophy limited to the duodenal bulb. J Clin Gastroenterol. 2007;41:571-5. doi: 10.1097/01. mcg.0000225625.99415.c0.

12. Bonamico M, Mariani P, Thanasi E, Ferri M, Nenna R, Tiberti C, et al. Patchy villous atrophy of the duodenum in childhood celiac disease. J Pediatr Gastroenterol Nutr. 2004;38:204-7. doi: 10.1097/00005176-200402000-00019.

13. Rodrigo L. Celiac disease. World J Gastroenterol. 2006;12:6585-93. doi: 10.3748/wjg.v12.i41.6585.

14. Mauriño E, Capizzano H, Niveloni S, Kogan Z, Valero J, Boerr $\mathrm{L}$, et al. Value of endoscopic markers in celiac disease. Dig Dis 
Sci. 1993;38:2028-33. doi: 10.1007/BF01297080.

15. Brocchi E, Tomassetti P, Misitano B, Epifanio G, Corinaldesi R, Bonvicini $F$, et al. Endoscopic markers in adult coeliac disease. Dig Liver Dis. 2002;34:177-82. doi: 10.1016/s15908658(02)80190-6.

16. Cammarota G, Fedeli P, Gasbarrini A. Emerging technologies in upper gastrointestinal endoscopy and celiac disease. Nat Clin Pract Gastroenterol Hepatol. 2009;6:47-56. doi: 10.1038/ ncpgasthep1298.

17. Volta U, Villanacci V. Celiac disease: diagnostic criteria in progress. Cell Mol Immunol. 2011;8:96-102. doi: 10.1038/ cmi.2010.64.

18. Dickey W, McMillan SA. Increasing numbers at a specialist coeliac clinic: contribution of serological testing in primary care. Dig Liver Dis. 2005;37:928-33. doi: 10.1016/j. dld.2005.07.011.

19. Dickey W. Endoscopic markers for celiac disease. Nat Clin Pract Gastroenterol Hepatol. 2006;3:546-51. doi: 10.1038/ ncpgasthep0601.

20. Zhou T, Han G, Li BN, Lin Z, Ciaccio EJ, Green PH, et al. Quantitative analysis of patients with celiac disease by video capsule endoscopy: A deep learning method. Comput Biol Med. 2017;85:1-6. doi: 10.1016/j.compbiomed.2017.03.031.

21. Black DD, Haggitt RC, Whitington PF. Gastroduodenal endoscopichistologic correlation in pediatric patients. J Pediatr Gastroenterol Nutr. 1988;7:353-8. doi: 10.1097/00005176198805000-00007.

22. Lombardi G, de' Angelis G, Rutigliano V, Guariso G, Romano C, Falchetti D, et al. Reflux oesophagitis in children; the role of endoscopy. A multicentric Italian survey. Dig Liver Dis. 2007;39:864-71. doi: 10.1016/j.dld.2007.05.018.

23. Oderda G, Forni M, Farina L, Dell'Olio D, Ansaldi N. Duodenitis in children: clinical, endoscopic, and pathological aspects. Gastrointest Endosc. 1987;33:366-9. doi: 10.1016/ s0016-5107(87)71640-x. 